\title{
Diagnostic Accuracy of Diffusion Weighted Imaging and Dynamic Imaging Techniques in Endometrial and Lymph Nodes Cancer Staging
}

\author{
Seyed Abkenari S.K. ${ }^{1}$ BSc, Faeghi F.* PhD, Arian A. ${ }^{2}$ PhD \\ *Radiology Technology Department, Allied Medical Sciences School, \\ Shahid Beheshti University of Medical Sciences, Tehran, Iran \\ 1Radiology Technology Department, Allied Medical Sciences School, \\ Shahid Beheshti University of Medical Sciences, Tehran, Iran \\ 2Imaging Center of Imam Khomeini Hospital, Tehran University of Medical \\ Sciences, Tehran, Iran
}

\begin{abstract}
Aims: Endometrial cancer is the most common malignancy of the female reproductive tract. As the prognosis factors, the determination of exact cancer stage and the differentiation of the lymph nodes plays a role in the selection of treatment plan and the rate of patient's survival. The aim of this study was to investigate the diagnosis accuracy of dynamic and diffusion imaging techniques in the endometrial cancer staging as well as the differentiation of metastatic and non-metastatic lymph nodes.

Materials \& Methods: In this prospective study, 30 patients with endometrial cancer were studied in MRI ward of the imaging center of Imam Khomeini Hospital in Tehran in 2013-14. The subjects, selected by purposeful sampling method, underwent the pelvis imaging before the surgery. $\mathrm{T}_{1} \mathrm{~W}$ and $\mathrm{T}_{2} \mathrm{~W}$ scenes, the dynamic technique in phases including $25,60,120$, and 240 seconds after the injection, and the diffusion technique $\left(b=\right.$ zero and $\left.1000 \mathrm{~s} / \mathrm{mm}^{2}\right)$ were used in the imaging. Data was analyzed by SPSS 22 software using kappa statistic, two-independent $\mathrm{T}$ test, and one-way ANOVA.

Findings: The agreement rate between the results of radiology and pathology in the determination of the different cancer stages was approximately 0.8 , showing a good and considerable result $(\mathrm{p}<0.001)$. The diagnostic authenticity rate of the dynamic and diffusion techniques was 0.83 . The results of radiology and pathology were completely consistent regarding the differentiation between the metastatic and non-metastatic lymph nodes. In different cancer stages, as well as in the metastatic and non-metastatic lymph nodes, the mean age and number of fertility were not significantly different ( $p>0.05)$.

Conclusion: In the differentiation between the metastatic and non-metastatic lymph nodes in patients with endometrial cancer, the diagnostic accuracy is increased by the diffusion techniques utilizing the routine scenes and the injection.
\end{abstract}

Keywords

Diffusion Magnetic Imaging

[http://www.ncbi.nlm.nih.gov/mesh/68038524];

Technetium Tc 99m Exametazime [http://www.ncbi.nlm.nih.gov/mesh/68019690];

Endometrial Neoplasms [http://www.ncbi.nlm.nih.gov/mesh/68016889];

Lymph Nodes [http://www.ncbi.nlm.nih.gov/mesh/68008198]

* Corresponding Author

Tel: +982122718531

Fax: +982122521170

Address: Radiology Technology Department, School of Allied Medical Sciences, Shahid Beheshti University of

Medical Sciences, Darband Street, Tehran, Iran

f_faeghi@sbmu.ac.ir

Received: July 20, 2015 Accepted: May 10, 2016 ePublished: June 30, 2016 


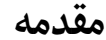

سرطان آندومتر جهارمين بدخيمى شايع در زنان و شايعترين

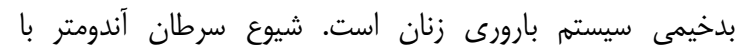

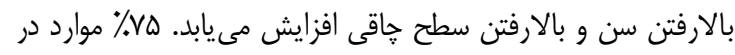

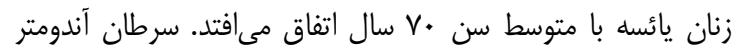

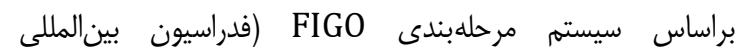

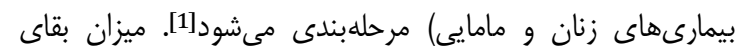

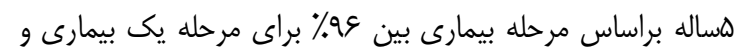

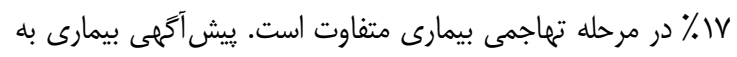

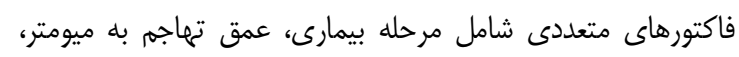

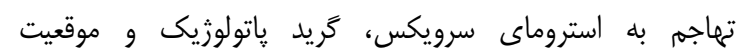

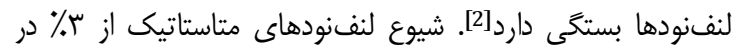

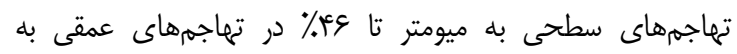

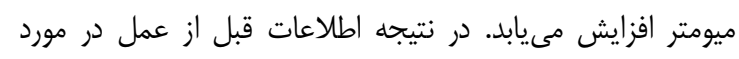

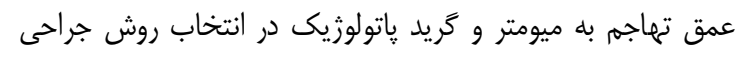
براى اين بيماران اساسى است [1].

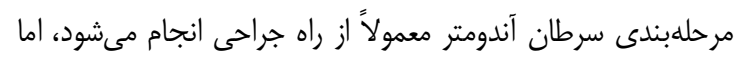

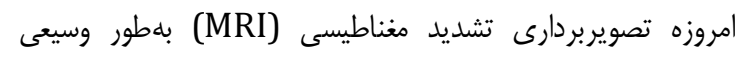

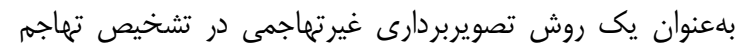

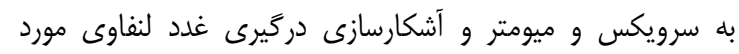

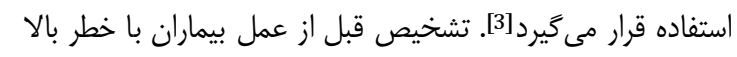

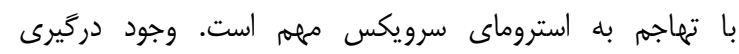
سرويكس قبل از عمل بلهوسيله يروسه كورتاز اندوسرويكس انجام انهام

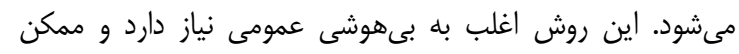

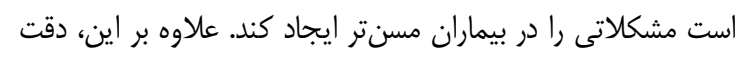

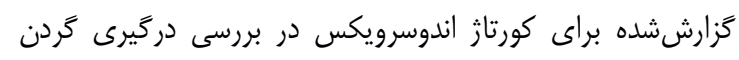

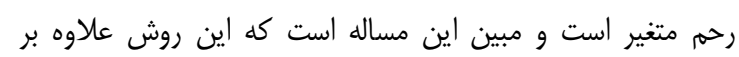

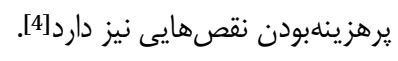

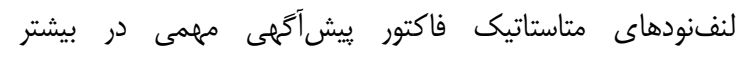

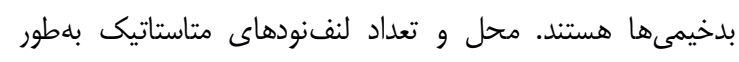

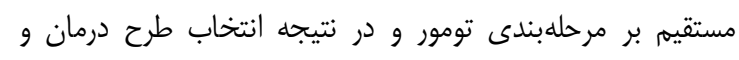

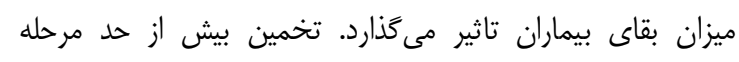

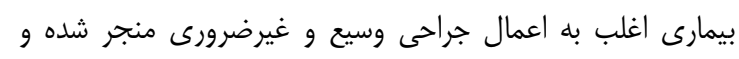

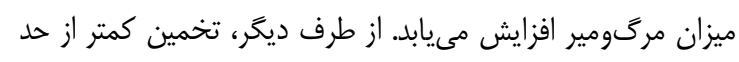

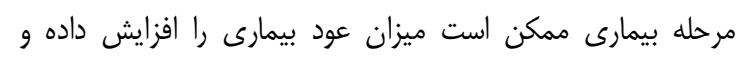

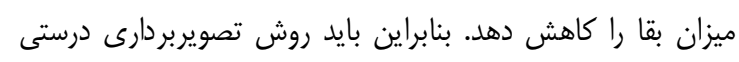

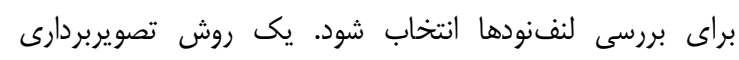
ايدهآل بايد قادر باشد محل و تغييرات ساختارى لنفنودها لنفا را آشكار

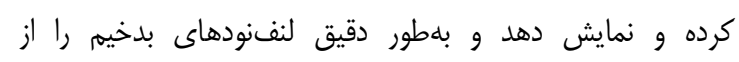

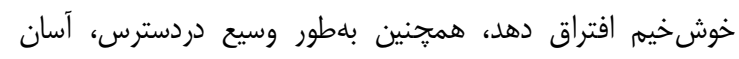

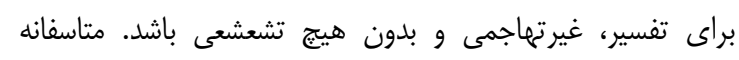

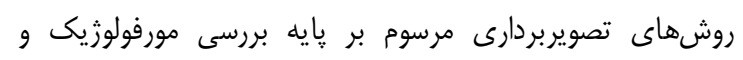

\section{دقت تشخيصى تكنيكهاى تصويريريردارى ديفيوزنى و ديناميكى در مرحلدبندى سرطين آندومتر و كَههاى لنفاوى دينى دينى}

سيده كبرى سيدآبكنارى BSc كروه تكنولوزى راديولوزى، دانشكده بيريرايزشكى، دانشكاه علوم يزشكى شهيد بهشتى، تهران، ايران

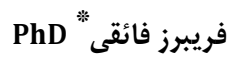
كروه تكنولوزى راديولوزى، دانشكده بيرايزشكى، دانشكاه علوم يزشكى شهيد بهشتى، تهران، ايران آروين آريان PhD بخش تصويربدارى بيمارستان امام خمينى، دانشكاه علوم يزشكى تهران، تهران، ايران

اهداف: سرطان آندومتر شايعترين بدخيمى مربوط به سيستم بارورى

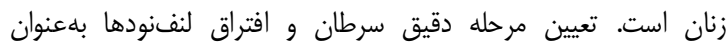

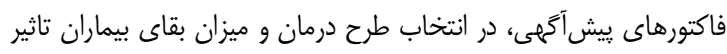

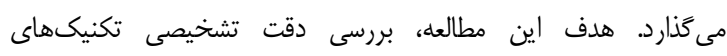

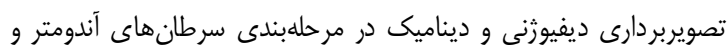

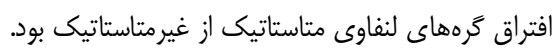

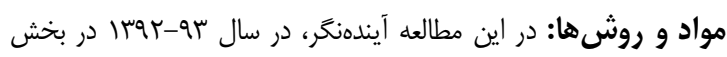

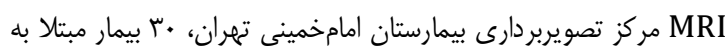

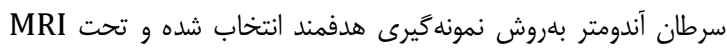

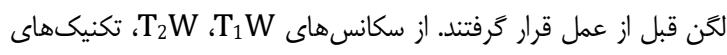

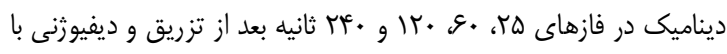

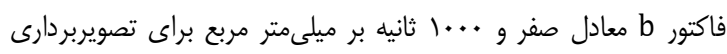

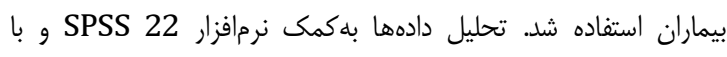

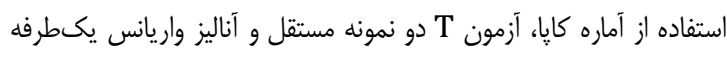
انجام شد. يافتهها: توافق بين نتايج راديولوزى و وِاتولوزى در تعيين مراحل مختلف

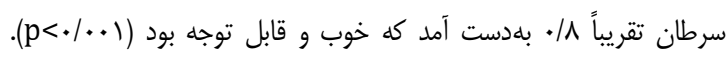

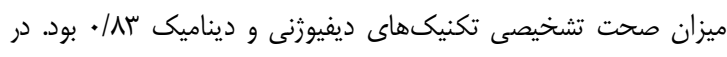

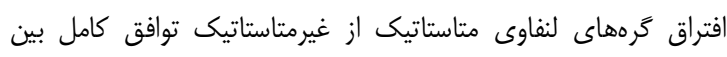

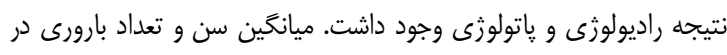

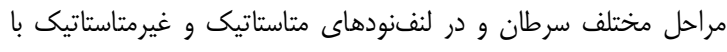

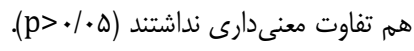

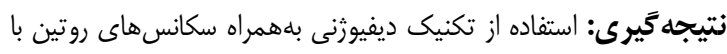

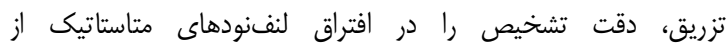

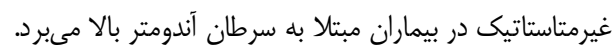

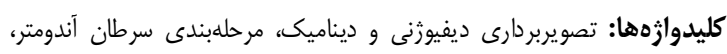
كرهاى لنفاوى

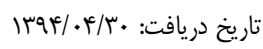

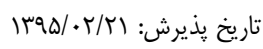
f_faeghi@sbmu.ac.ir :نويسنده مسئول:

دوره זr، شماره ז، تابستان هوبr

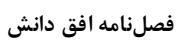


PAD دقت تشخيصى تكنيكهاى تصويربردارى ديفيورنى و ديناميكى در مرحلدبندى سرطان آندومتر و ترههاى لنفاوى

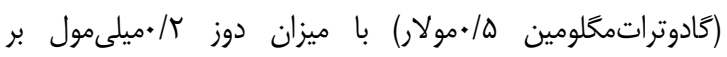

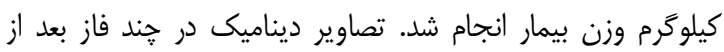

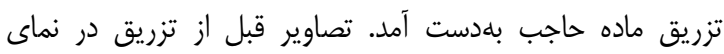

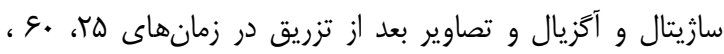

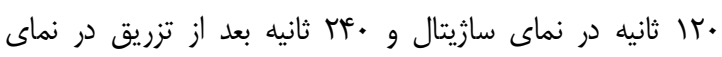
اگزيال گرفته شد. اين تصاوير توسط راديولوزيست با سابقه تجربه در زمينه

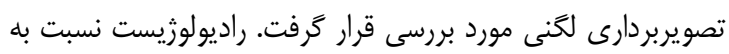

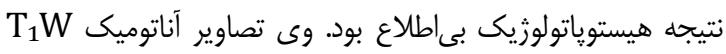

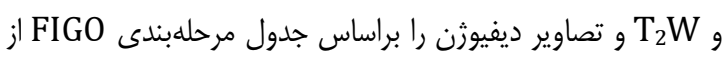

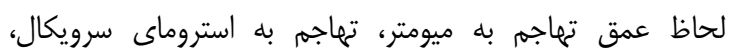

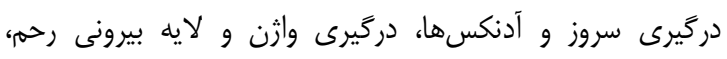

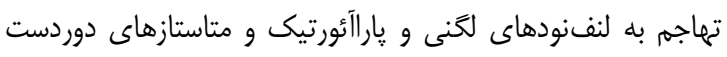

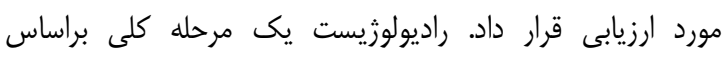

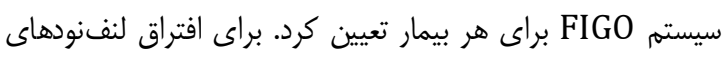

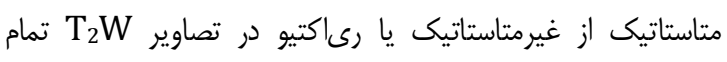

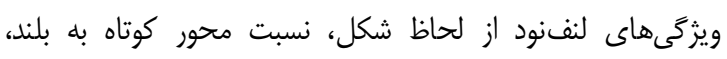

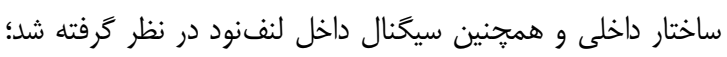

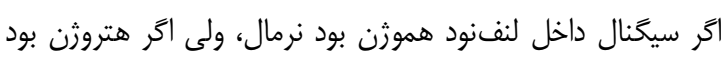

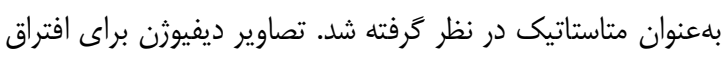

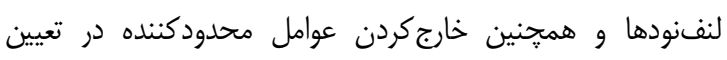

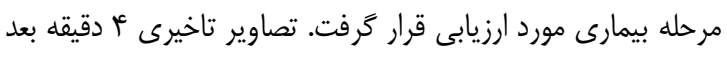

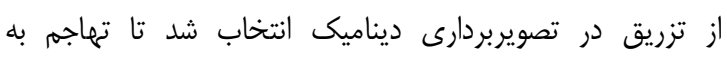
سرويكس بلهور دقيقتر بررسى شود.

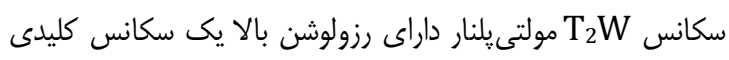

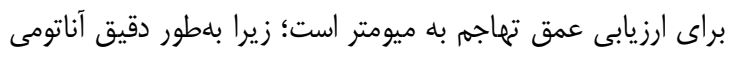

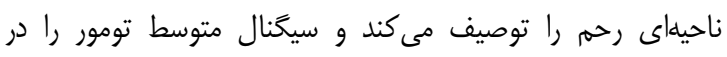

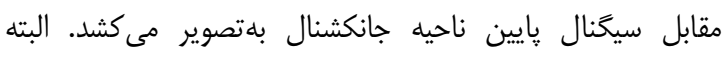

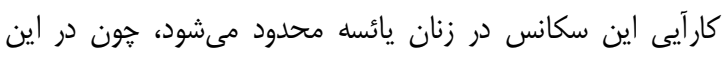

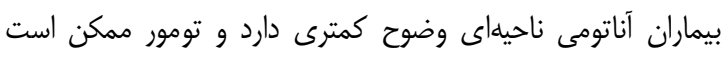

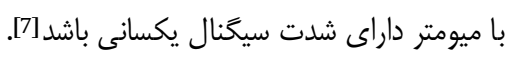

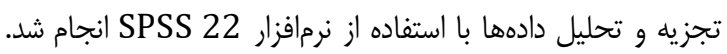

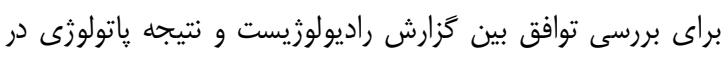

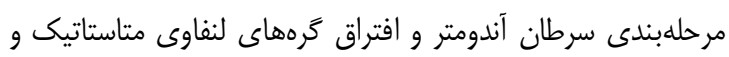

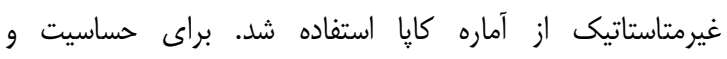

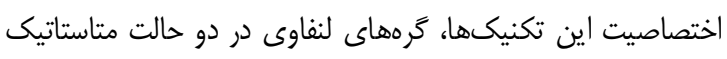

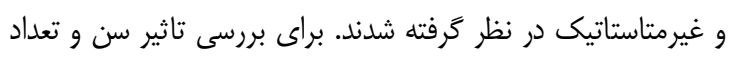

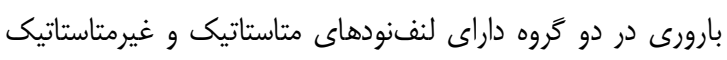

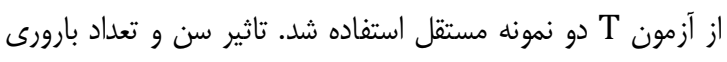
در مراحل مختلف سرطان نيز با استفاده از آزمون آناليز واريانس ندانس يكسرفه مورد بررسى قرار ترفت.

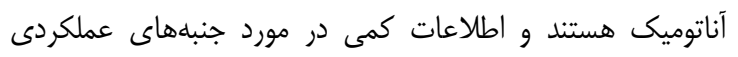

لنفنودها فراهم مى كنند [5].

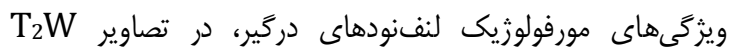

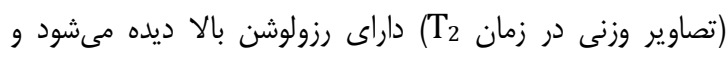

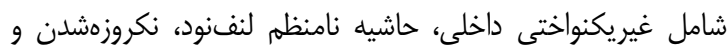

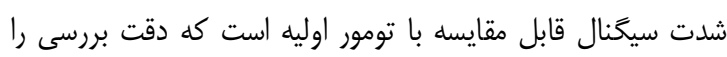

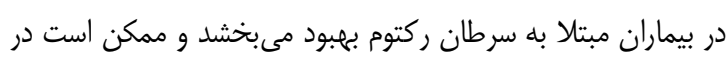

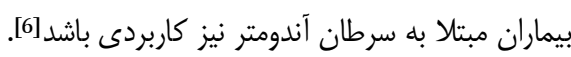

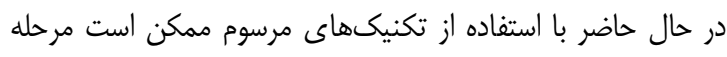

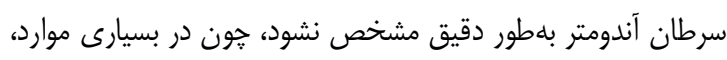

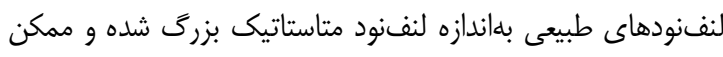

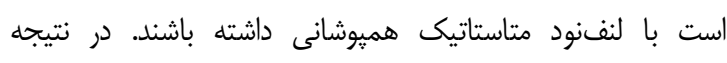

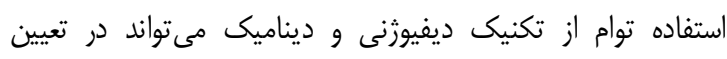

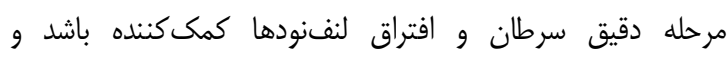

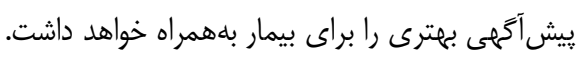

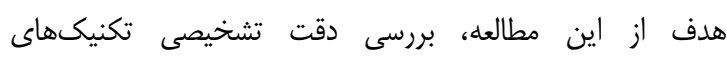

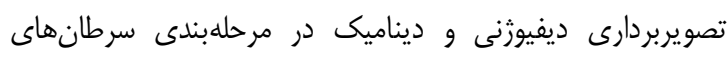

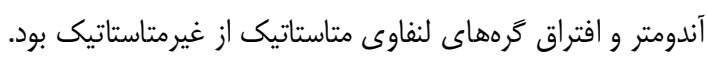

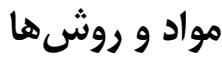

در اين مطالعه آيندهنكر كه از مهر سال זوحسا تا مهر سال سوسا در بخش MRI مركز تصويربردارى بيمارستان امامخمينى تهران انجام

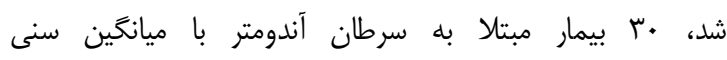

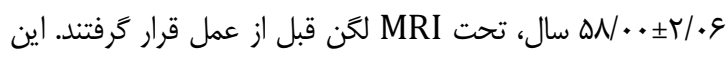

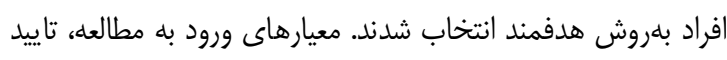

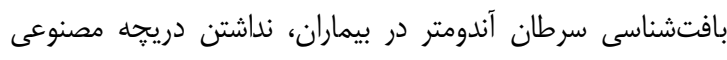

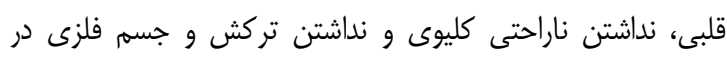

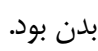

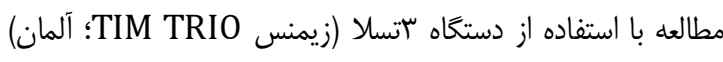

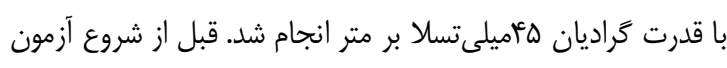

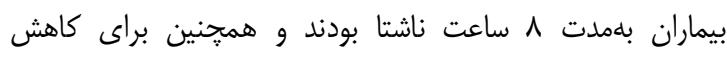

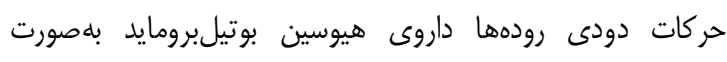

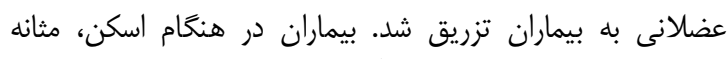

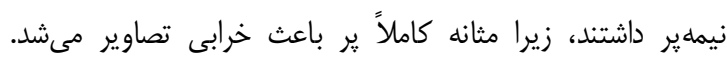

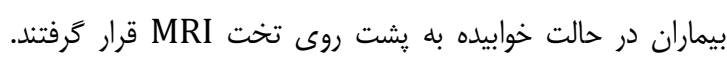

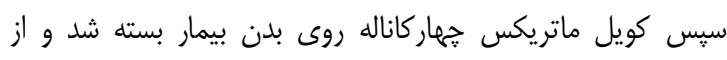

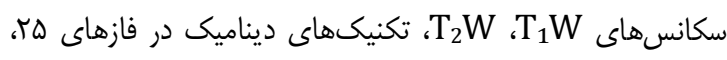

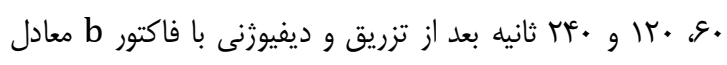

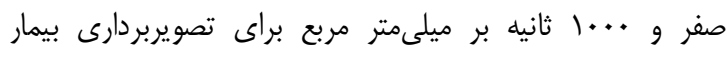

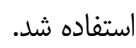
تصويربردارى ديناميك لكن بعد از تزريق ماده كنتراست دوتارم 


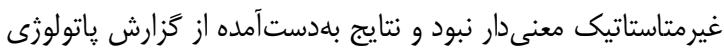

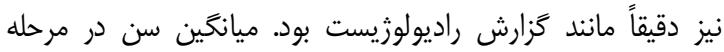
كمترين و در مرحله IIIC2 IIIA كمداد بارورى نيز در مرحله IIIC2

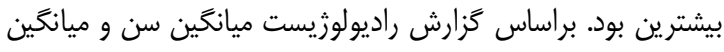

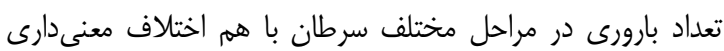

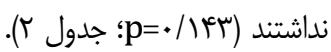

بحث

يشيش آحمى بيماران مبتلا به سرطان آندومتر به فاكتورهاى زيادى از

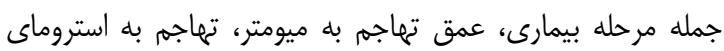

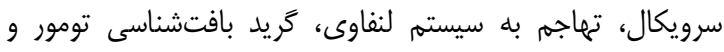

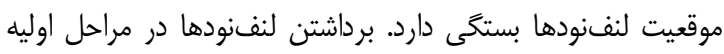

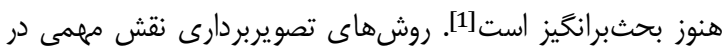

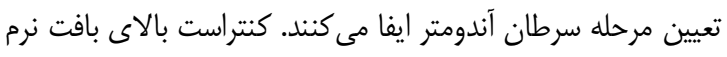

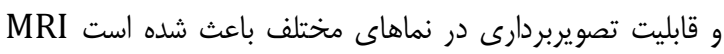

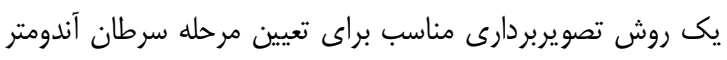

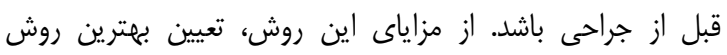

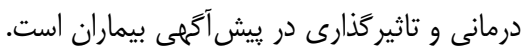

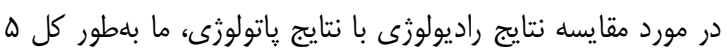

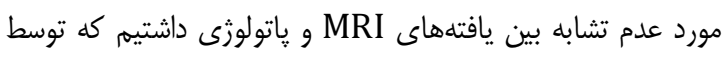

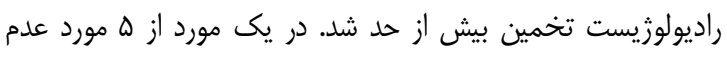

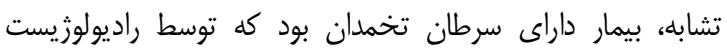

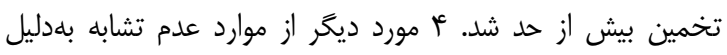

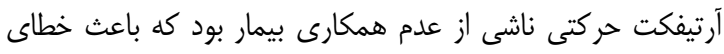

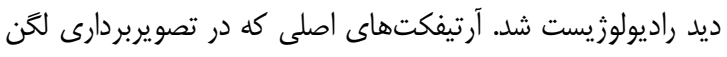

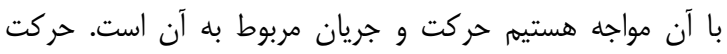

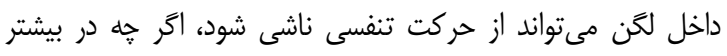

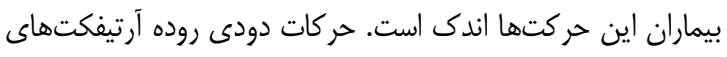

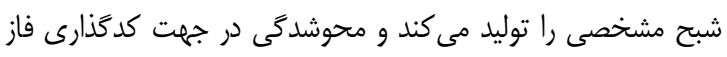

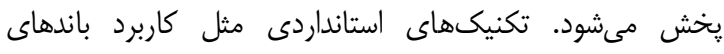

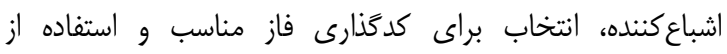

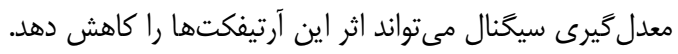

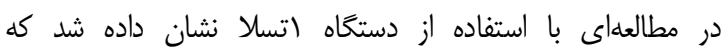

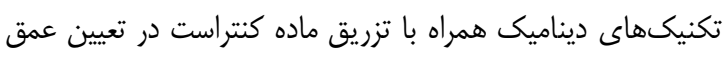

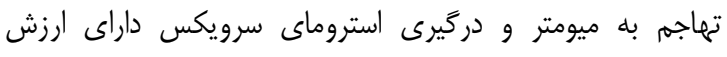

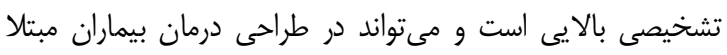

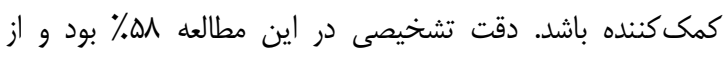

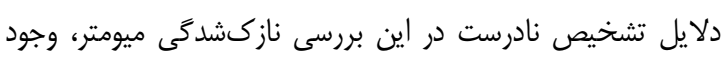

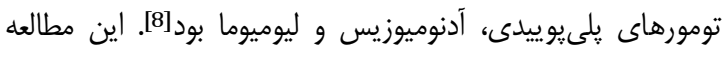

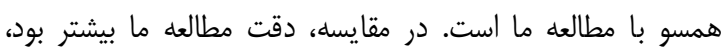
بهدليل اينكه علاوه بر تكنيكهاى روتين از تكنيك ديفيوزنى نيز
يافتهها

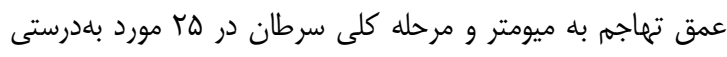

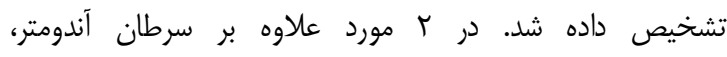

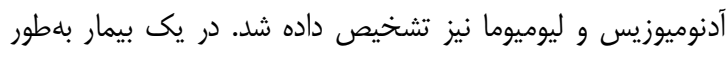

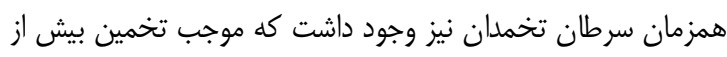
اندازه مرحله سرطان توسط راديولوزيست شدان نيز

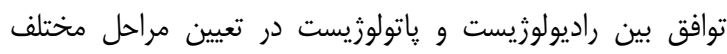

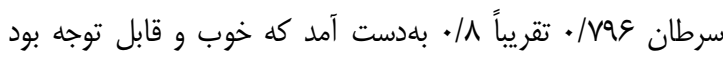

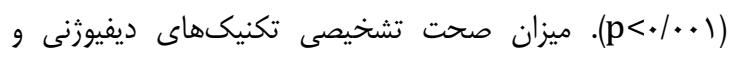

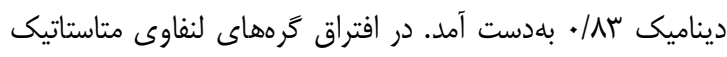

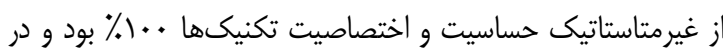

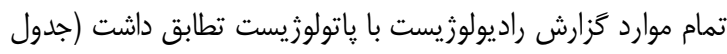

جدول () جدول توافقى بين كزارش راديولوزيست و باتولوثى در مورد مراحل سرطان

\begin{tabular}{|c|c|c|c|c|c|c|c|c|}
\hline كل & IIIC2 & IIIC1 & IIIB & IIIA & II & IB & IA & 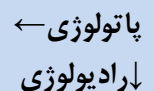 \\
\hline v & . & . &. & . & $\cdot$ & . & $v$ & IA \\
\hline v & . & $\cdot$ & . & . & . & r & $r$ & IB \\
\hline r & . & $\cdot$ & . & . & r & . & 1 & II \\
\hline r & . & · & . & $r$ & $\cdot$ & $\cdot$ & 1 & IIIA \\
\hline 1 & . & . & 1 & . & . & . & . & IIIB \\
\hline$\Delta$ & . & Q & . & . & . & $\cdot$ & . & IIIC1 \\
\hline r & ${ }^{r}$ & $\cdot$ & . & . & $\cdot$ & $\cdot$ & . & IIIC2 \\
\hline r. & r & a & 1 & $r$ & $r$ & r & ir & كل \\
\hline
\end{tabular}

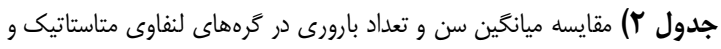

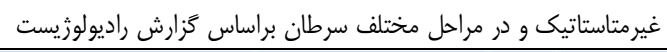

\begin{tabular}{|c|c|c|c|}
\hline ميانكين تعداد بارورى & ميانكين سن (سال) & تعداد & متغيرها \\
\hline & & \multicolumn{2}{|c|}{ كرههاى لنفاوى } \\
\hline$r / V \Delta \pm \cdot / V$ & $\Delta V / I r \pm r / q V$ & $\wedge$ & متاستاتيك \\
\hline \multirow[t]{2}{*}{$F / Q \Delta \pm \cdot / \Delta \Lambda$} & $\Delta N / F I \pm T / \Psi \varepsilon$ & tr & غيرمتاستاتيك \\
\hline & \multicolumn{3}{|c|}{ مر احل مختلف سرطان } \\
\hline$p / \ldots \pm \cdot / 4 q$ & $\Delta r / \wedge \varepsilon_{ \pm} / \wedge V$ & v & IA \\
\hline$r / \Delta V \pm \cdot / 9 q$ & $\Delta Q / \cdots \pm \Psi / / V$ & $\checkmark$ & IB \\
\hline$\Delta / \Psi_{ \pm} \cdot / \& V$ & $\varepsilon V / \cdot \cdot \pm \varepsilon / \cdot \Lambda$ & r & II \\
\hline$V / \cdots \pm r / \wedge q$ & $\Phi \mu / \& \mathrm{~V} \pm \mathrm{N} / \mathrm{r}$ & r & IIIA \\
\hline$\varphi / \cdots \pm 1 / q \mu$ & $\Delta V / \cdots \pm \varepsilon / \mu \wedge$ & 1 & IIIB \\
\hline$\varepsilon / 9 \cdot \pm \cdot|\wedge|$ & $9 \Delta / 9 \cdot \pm \Delta / Q V$ & $\Delta$ & IIIC1 \\
\hline$r / r \Delta \pm 1 / T \Delta$ & $F N / T \Delta \pm T / F q$ & r & IIIC2 \\
\hline$r / q \cdot \pm \cdot / 4 \varepsilon$ & $\Delta N / \cdot V \pm r / \cdot G$ & $r$. & كل \\
\hline
\end{tabular}

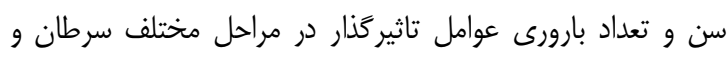

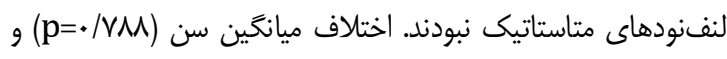

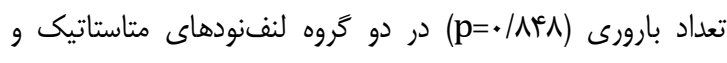

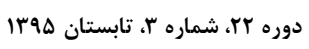
فصلنامه افق دانش 


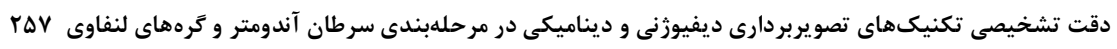

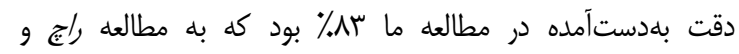

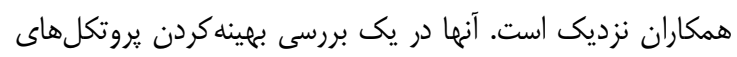

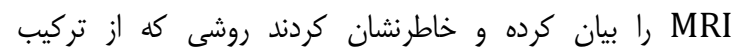
سكانسهاى آناتوميكال Th

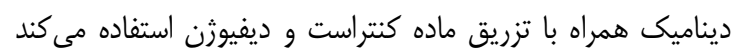

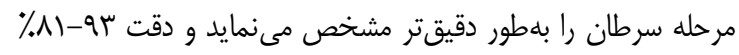

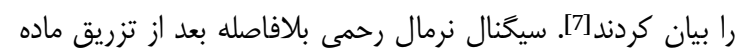

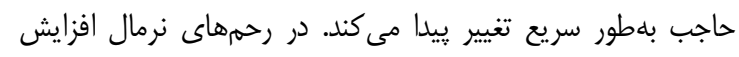

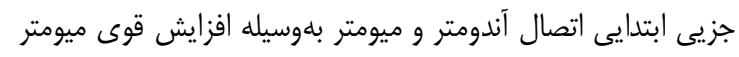

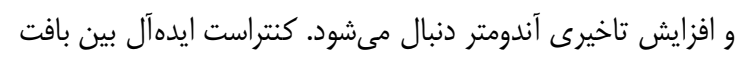

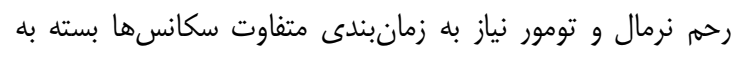

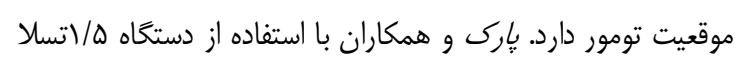

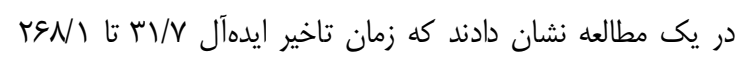

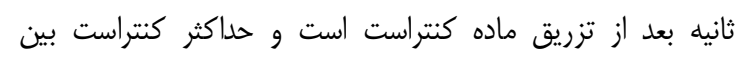

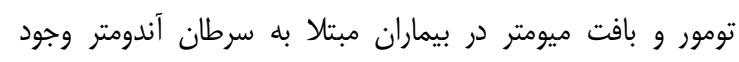

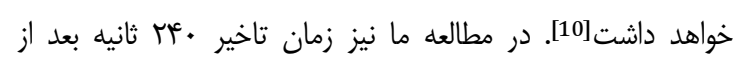

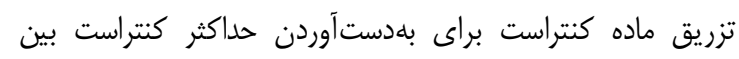

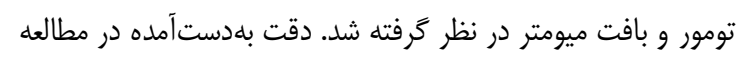

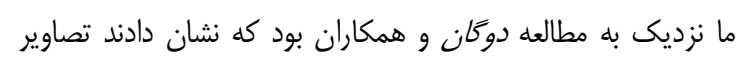

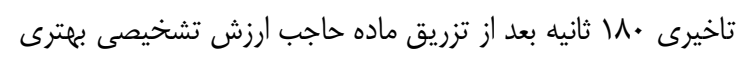

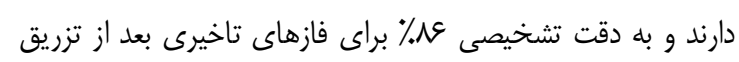

ماده كنتراست دست بيدا كردند [111].

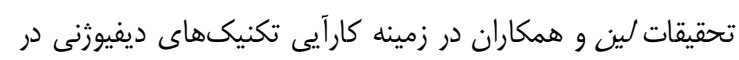
تاييد مطالعه ما نشان داده كه تركيب سكانس

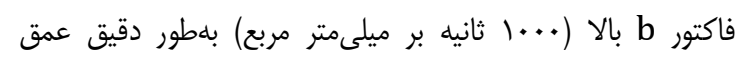

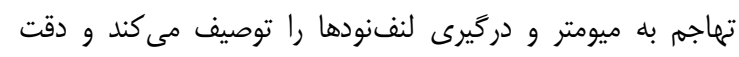

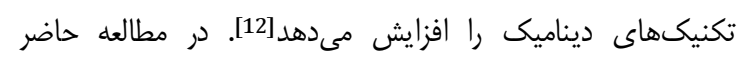

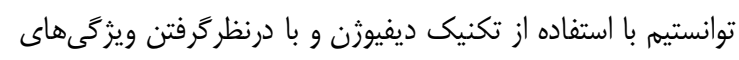

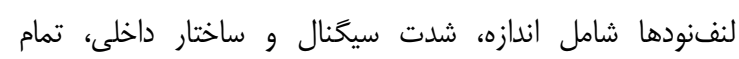

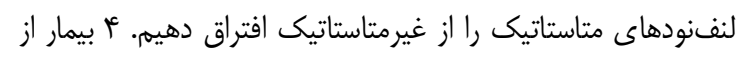

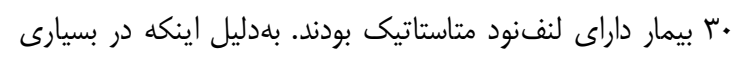

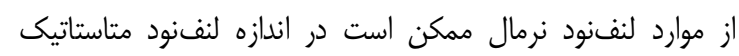

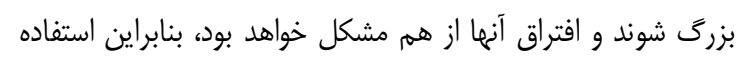

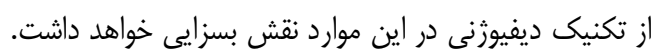

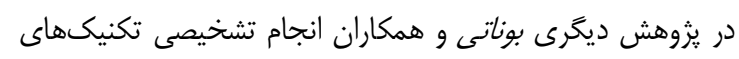

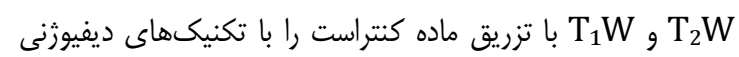

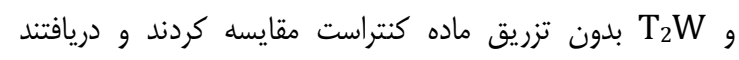

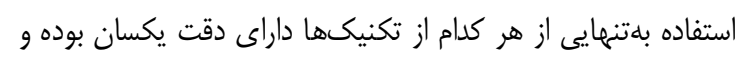

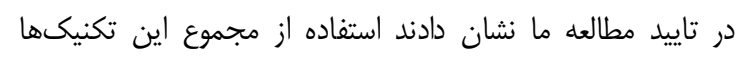

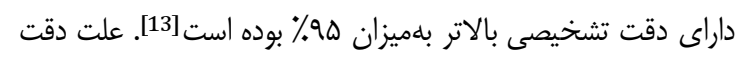
بيشتر مطالعه آنها تعداد بيشتر بيماران نسبت بهان به مطالعه ما بود. نتايج
استفاده شد و توانستيم اين موارد خوشخيه را از سرطان آندومتر

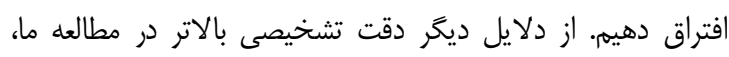

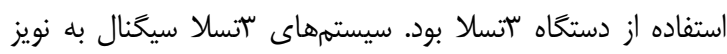

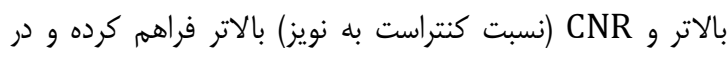

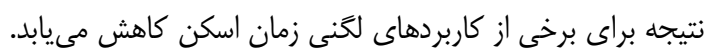

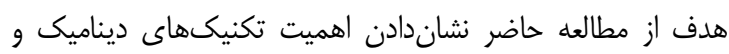

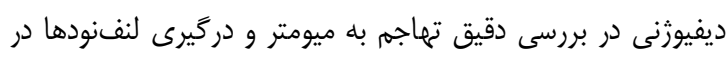

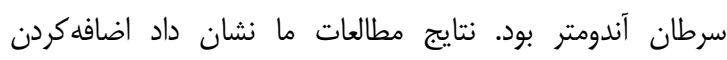
تكنيكهاى ديفيوزنى و ديناميك به تلكنيكهان

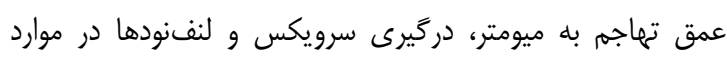

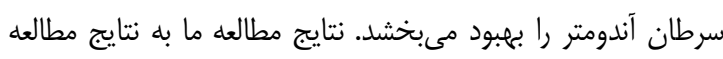

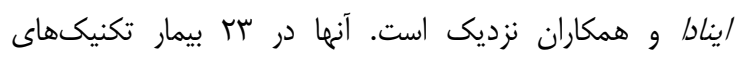

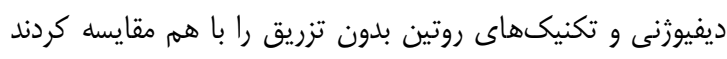

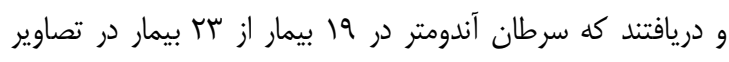

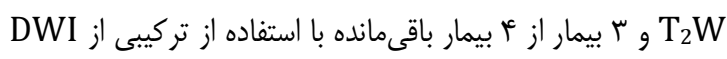

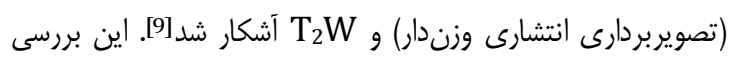

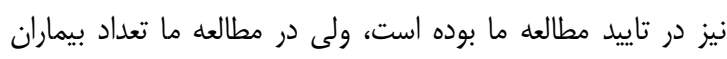

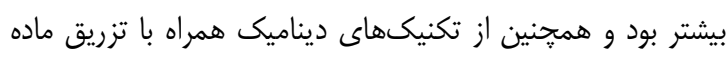

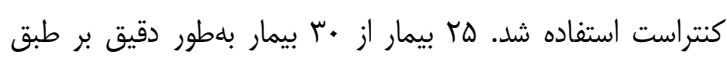
نتيجه پِاتولوزى مرحلهبندى شدند. سكانسهاى T2W آناتومى احشاى لكن ران ران نشان مى دهند و تقريباً

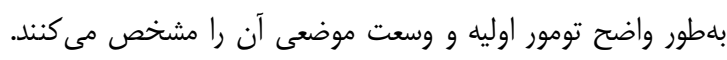

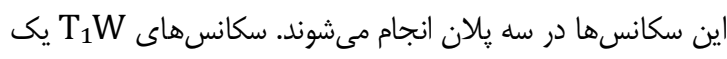

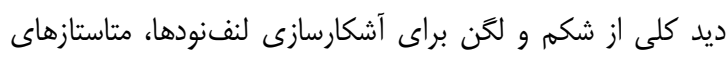

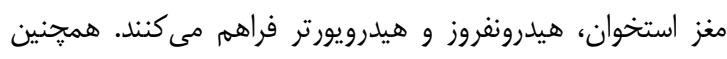

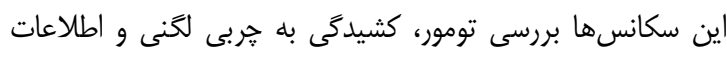

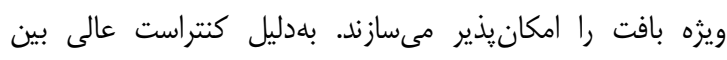

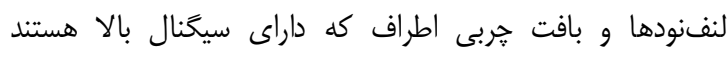

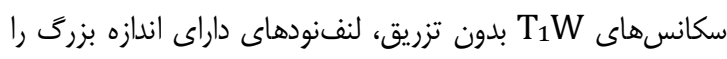
خيلى سريعتر نسبت به سكانسهاى Th

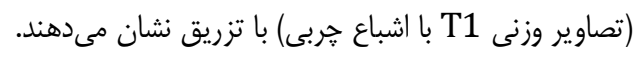

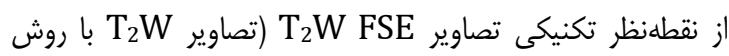

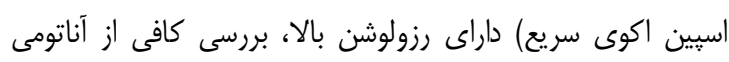

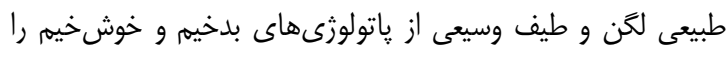

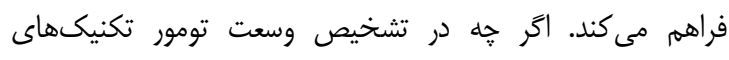

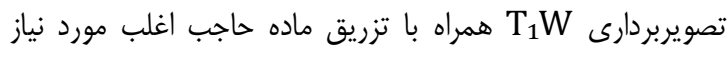

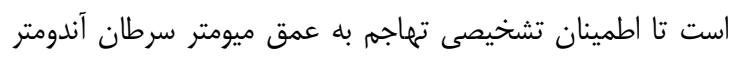

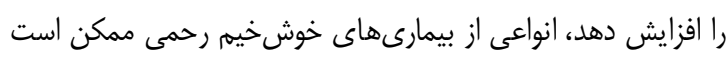

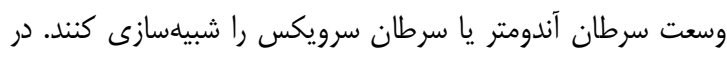

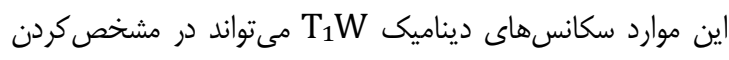
لندماركهاى آناتوميك مانند منطقه جانكشنال كمك دكننده باشدان 


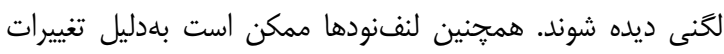

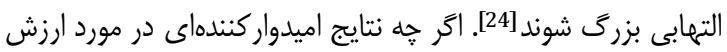

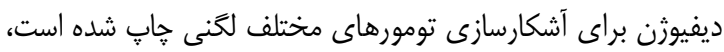
هنوز مطالعات محدودى در مورد بالقوهبودن تكنيك ديفيوزنى براي افتراق لنفنودها وجود دارد. بنابراين تركيب دو تكنيك ديناميك و دوردات

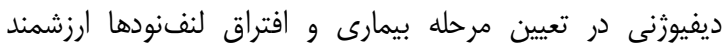

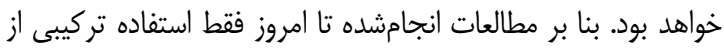

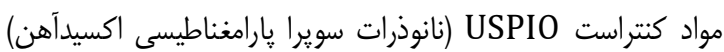
و تكنيكهاى روتين MRI بهترين نتايج را براى آشكارسازى

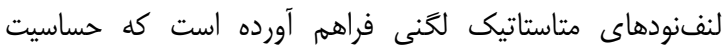
كزارش شده براى آن ... \% بوده است [25]. نتايج تروهش تئونى و همكاران نشان داد كه تركيب تكنيكهاى

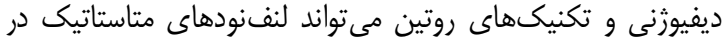

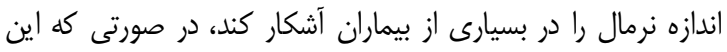

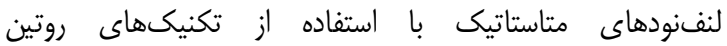
سىتىاسكن و MRI تشخيص داده نشده بودند [26]. حساسيت و اختصاصيت اين تكنيكها در افتراق لنفنودها بر طبق اين مطالعه

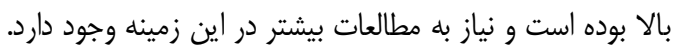

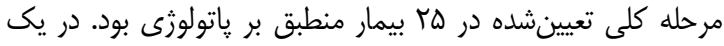

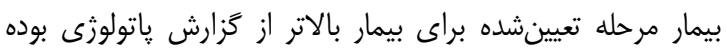

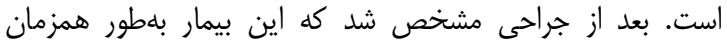

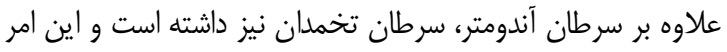

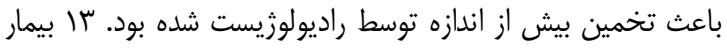

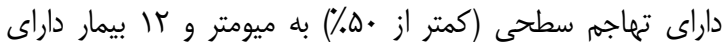

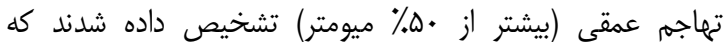

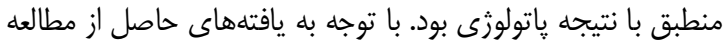

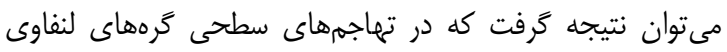

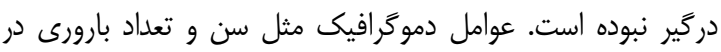
ايجاد سرطان آندومتر و متاستاز لنفنودها تاثيرى نداشتند.

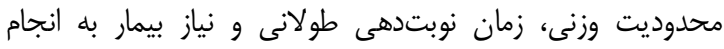

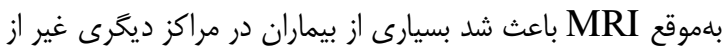
مركز تعيينشده MRI خود را انجام داده و در نتيجه از مطالعه دئه

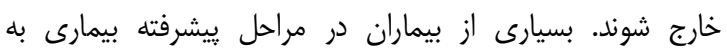

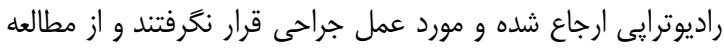

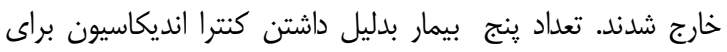

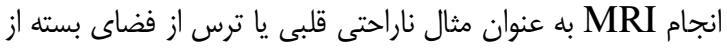

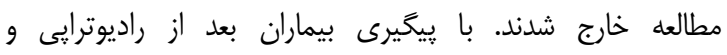

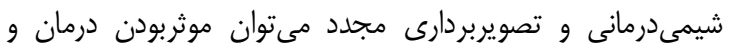

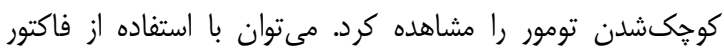
ADC

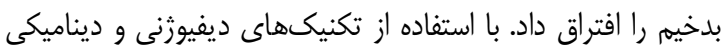
مى توان تغييرات بعد از درمان را از عود مجدد تومور افتراق داد.

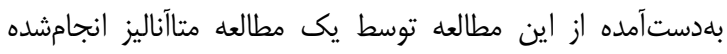
توسط داس و همكاران تاييد شده است [14].

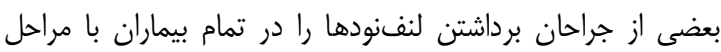

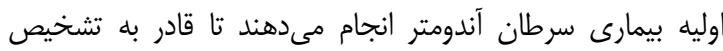

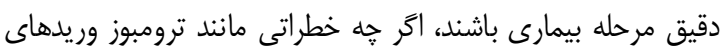

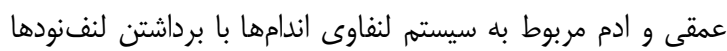

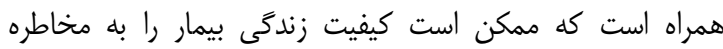

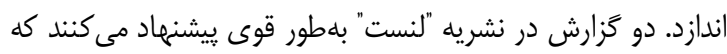

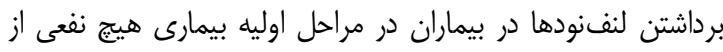

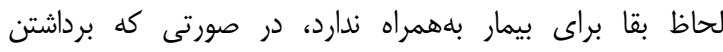
لنفنودها در بيماران با مراحل متوسط و ييشرفته بيمارى بقاى بيمار

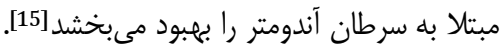

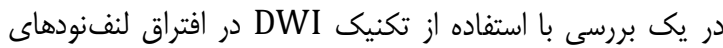

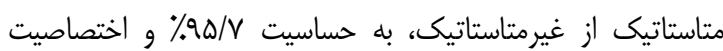

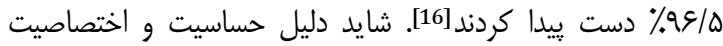
بإيين در اين مطالعه نسبت به مطالعه ما عدم استفاده از تكنيكهاي با تزريق بوده است. مطالعات نشان دادهاند كه سرطان آندومتر بهطور مشخصى ضريب

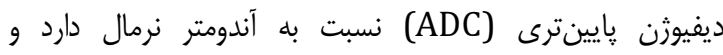

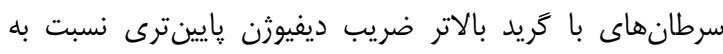

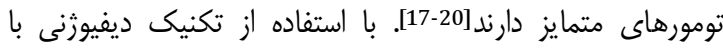

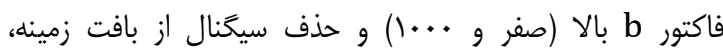

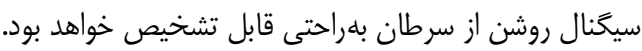

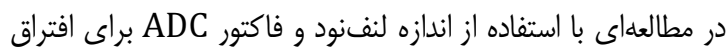

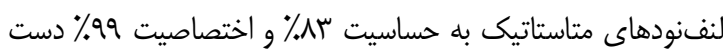

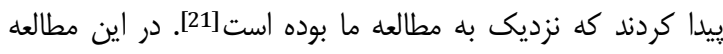

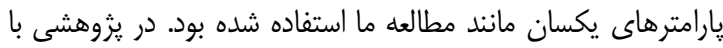

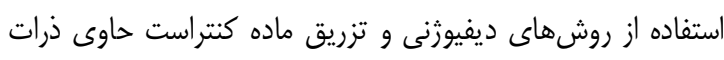

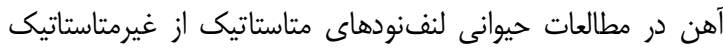

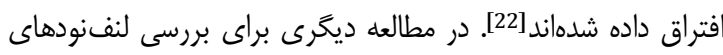

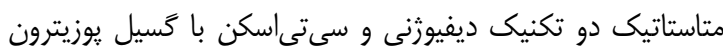

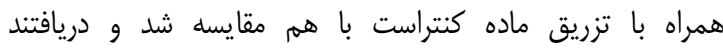

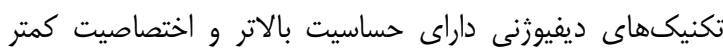

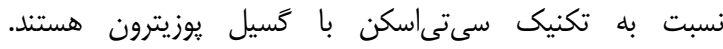

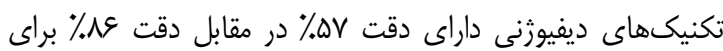
تكنيكهاى با كسيل يوزيترون بود[23].

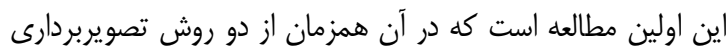

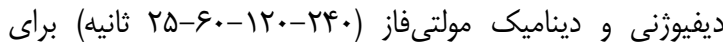

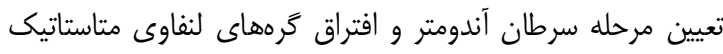

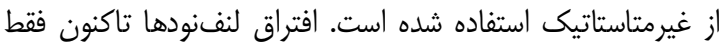

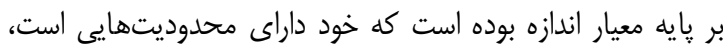
زيرا ميكرومتاستازها نيز ممكن است در اندازه لنفنودهاى نرمال 
دقت تشخيصى تكنيكهاى تصويربردارى ديفيوزنى و ديناميكى در مرحلهبندى سرطان آندومتر و ترههاى لنفاوى

and Cervical Cancer. Radiogra. 2014;34(4):1082-98.

8- Sanjuan A, Escaramis G, Ayuso JR, Roman SM, Torne A, Ordi J, et al. Role of magnetic resonance imaging and cause of pitfalls in detecting myometrial invasion and cervical involvement in endometrial cancer. Arch Gynecol Obstet. 2008;278(6):535-9.

9- Inada Y, Matsuki M, Nakai G, Tatsugami F, Tanikake M, Narabayashi I, et al. Body diffusion-weighted MR imaging of uterine endometrial cancer: Is it helpful in the detection of cancer in nonenhanced MR imaging?. Eur J Radiol. 2009;70(1):122-7.

10- Park SB, Moon MH, Sung CK, Oh S, Lee YH. Dynamic contrast-enhanced $\mathrm{mr}$ imaging of endometrial cancer: optimizing the imaging delay for tumour-myometrium contrast. Eur Radiol. 2014;24(11):2795-9.

11- Dogan D, Inan N, Sarisoy H, Gumustas S, Akansel G, Muezzınoğlu B, et al. Preoperative evaluation of myometrial invasion in endometrial carcinoma: diagnostic performance of 3T MRI. Abdom Imaging. 2013;38(2):388-96.

12- Lin G, Ng KK, Chang CJ, Wang JJ, Ho KC, Yen TC, et al. Myometrial invasion in endometrial cancer: Diagnostic accuracy of diffusion-weighted 3.0-T MR imaging-initial experience. Radiol. 2009;250(3):784-92.

13- Bonatti M, Stuefer J, Oberhofer N, Negri G, Tagliaferri T, Schifferle G, et al. MRI for local staging of endometrial carcinoma: Is endovenous contrast medium administration still needed?. Eur J Radiol. 2015;84(2):208-14

14- Das SK, Niu XK, Wang JL, Zeng LC, Wang WX, Bhetuwal A, et al. Usefulness of DWI in preoperative assessment of deep myometrial invasion in patients with endometrial carcinoma: A systematic review and metaanalysis. Cancer Imaging. 2014;14(1):32.

15- Mak S, Hulse PA, Carrington BM. MRI mnual of pelvic cancer. 2nd edition. Boca Raton, Florida: CRC Press; 2011. pp. 115-20.

16- Liu Y, Liu H, Bai X, Ye Z, Sun H, Bai R, et al. Differentiation of metastatic from non-metastatic lymph nodes in patients with uterine cervical cancer using diffusion-weighted imaging. Gynecol Oncol. 2011;122(1):19-24.

17- Fujii S, Matsusue E, Kigawa J, Sato S, Kanasaki Y, Nakanishi J, et al. Diagnostic accuracy of the apparent diffusion coefficient in differentiating benign from malignant uterine endometrial cavity lesions: Initial results. Eur Radiol.2008;18(2):384-9.

18- Levy A, Medjhoul A, Caramella C, Zareski E, Berges O, Chargari $C$, et al. Interest of diffusion-weighted echoplanar MR imaging and apparent diffusion coefficient mapping in gynecological malignancies: A review. J Magn Reson Imaging. 2011;33(5):1020-7.

19- Motoshima S, Irie H, Nakazono T, Kamura T, Kudo S. Diffusion-weighted MR imaging in gynecologic cancers. J Gynecol Oncol. 2011;22(4):275-87.

20-Tamai K, Koyama T, Saga T, Umeoka S, Mikami Y, Fujii $S$, et al. Diffusion-weighted MR imaging of uterine endometrial cancer. J Magn Reson Imaging. 2007;26(3):682-7.

21- Lin G, Ho KC, Wang JJ, Ng KK, Wai YY, Chen YT, et al. Detection of lymph node metastasis in cervical and uterine cancers by diffusion-weighted magnetic resonance imaging at $3 \mathrm{~T}$. J Magn Reson Imaging. 2008;28(1):128-35.

22- Zhang F, Zhu L, Huang $X$, Niu G, Chen X. Differentiation of Reactive and Tumor Metastatic Lymph Nodes with Diffusion-weighted and SPIO-Enhanced MRI.

\section{نتيجه كيرى}

استفاده از تكنيك ديفيوزنى بههمراه سكانسهاى روتين با تزريق،

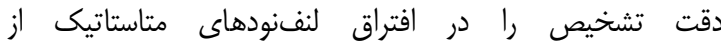

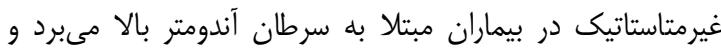

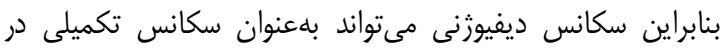

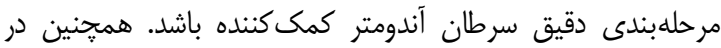
مواردى كه بيمار به راديوترايى ارجاع داده مىشود با توجه به به مرحله

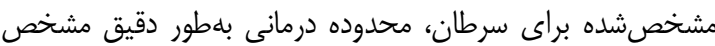

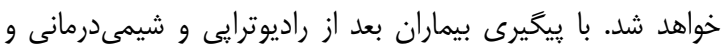

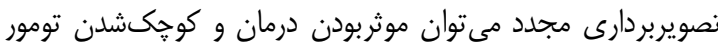
راهداهد كرد.

$$
\begin{aligned}
& \text { تشكر و قدردانى: اين مقاله برگرفته از پايانامه كارشناسىارشد }
\end{aligned}
$$

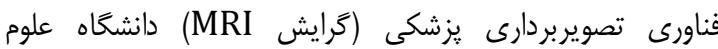

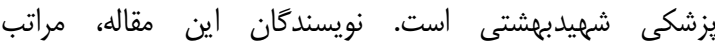

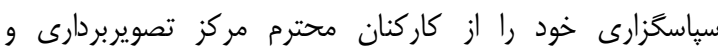

$$
\begin{aligned}
& \text { درمانگاه زنان بيمارستان امامخمينى تهران ابراز مى دارند. } \\
& \text { تاييديه اخلاقى: انجام اين يُوهش توسط كميته اخلاق دانشگاه }
\end{aligned}
$$

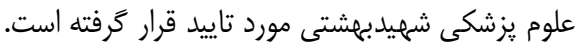

$$
\begin{aligned}
& \text { تعارض منافع: هيج گَونه تعارض منافع توسط نويسندكان بيان } \\
& \text { شده است. } \\
& \text { حمايت مالى: اين مقاله حاصل پاياننامه كارشناسىارشد دانشگًاه } \\
& \text { علوم يزشكى شهيدبهشتى است. } \\
& \text { منابع }
\end{aligned}
$$

1- Beddy P, O'Neill AC, Yamamoto AK, Addley HC, Reinhold C, Sala E. FIGO staging system for endometrial cancer: Added benefits of MR imaging. Radiogr. 2012;32(1):241-54.

2- Beddy P, Moyle P, Kataoka M, Yamamoto AK, Joubert I, Lomas D, Crawford R, et al. Evaluation of depth of myometrial invasion and overall staging in endometrial cancer: Comparison of diffusion-weighted and dynamic contrast-enhanced MR imaging. Radiol. 2012;262(2):530-7.

3- Rha SE, Byun JY, Jung SE, Lee SL, Cho SM, Hwang SS, et al. CT and MRI of Uterine Sarcomas and Their Mimickers. Am J Roentgenol. 2003;181(5):1369-74.

4- Van den Bosch T, Coosemans A, Morina M, Timmerman D, Amant F. Screening for uterine tumours. Clin Obstet Gynaecol. 2012;26(2):257-66.

5- Larson DM, Connor GP, Broste SK, Krawisz BR, Johnson KK. Prognostic significance of gross myometrial invasion with endometrial cancer. Obstet Gynecol. 1996;88(3):394-8.

6- Kaur H, Choi H, You YN, Rauch GM, Jensen CT, Hou P, et al. MR Imaging for Preoperative Evaluation of Primary Rectal Cancer: Practical Considerations. Radiogr. 2012;32(2):389-409.

7- Rauch GM, Kaur H, Choi H, Ernst RD, Klopp AH, Boonsirikamchai $\mathrm{P}$, et al. Optimization of MR Imaging for Pretreatment Evaluation of Patients with Endometrial 
25- Harisinghani MG, Barentsz J, Hahn PF, Deserno WM, Tabatabaei S, van de Kaa $\mathrm{CH}$, et al. Noninvasive detection of clinically occult lymph-node metastases in prostate cancer. New Engl J Med. 2003;348(25):2491-9.

26- Thoeny HC, Froehlich JM, Triantafyllou M, Huesler J, Bains LJ, Vermathen P, et al. Metastases in normal-sized pelvic lymph nodes: Detection with diffusion-weighted MR imaging. Radiol. 2014;273(1):125-35.
ع ع سيده كبرى سيد آبكنارى و همكاران Mol Imaging Biol. 2013;15(1):40-7.

23- Kitajima K, Yamasaki E, Kaji Y, Murakami K, Sugimura K. Comparison of DWI and PET/CT in evaluation of lymph node metastasis in uterine cancer. World J Radiol. 2012;4(5)207-14.

24- Harriet C, Posemarie F, Frederik D. genitourinary applications of diffusion-weighted $\mathrm{mr}$ imaging in the pelvis. Radiol. 2012;263(2):326-42. 